\title{
Novel Sulfonamide Derivatives Carrying a Biologically Active 3,4-Dimethoxyphenyl Moiety as VEGFR-2 Inhibitors
}

\author{
Mostafa Mohammed Ghorab, ${ }^{*, a, b}$ Mansour Sulaiman Alsaid ${ }^{a}$ Yassin Mohammed Nissan, ${ }^{c}$ \\ Abdelkader Elbadawy Ashour, ${ }^{d}$ Abdullah Abdulalrahman Al-Mishari, ${ }^{e}$ Ashok Kumar, ${ }^{f}$ and \\ Sheikh Fayaz Ahmed ${ }^{d}$ \\ ${ }^{a}$ Department of Pharmacognosy, College of Pharmacy, King Saud University; P.O. Box 2457, Riyadh 11451, Saudi \\ Arabia: ${ }^{b}$ Department of Drug Radiation Research, National Center for Radiation Research and Technology; Nasr \\ City, P.O.Box 29, Cairo 11371, Egypt: ${ }^{c}$ Department of Pharmaceutical Chemistry, Faculty of Pharmacy, Cairo \\ University; Cairo, Egypt, El-Kasr El-Eini Street, Cairo 11562, Egypt: ${ }^{d}$ Department of Pharmacology and Toxicology, \\ College of Pharmacy, King Saud University; P.O. Box 2457, Riyadh 11451, Saudi Arabia: ${ }^{e}$ Medicinal, Aromatic and \\ Poisonous Plants Research Center (MAPPRC), College of Pharmacy, King Saud University; P.O. Box 2457, Riyadh \\ 11451, Saudi Arabia: and ${ }^{f}$ Vitiligo Research Chair, College of Medicine, King Saud University; Riyadh 11451, Saudi \\ Arabia. \\ Received July 31, 2016; accepted September 8, 2016
}

Novel sulfonamides 3-19 with a biologically active 3,4-dimethoxyphenyl moiety were designed and synthesized. The structures of the synthesized compounds were established using elemental analyses, IR, ${ }^{1} \mathrm{H}-\mathrm{NMR},{ }^{13} \mathrm{C}$-NMR spectral data and mass spectroscopy. All the synthesized compounds were evaluated for their in vitro anticancer activity against four cancer cell lines, namely human hepatocellular carcinoma (HepG2), human medulloblastoma (Daoy), human cervical cancer (HeLa), and human colon cancer (HT-29), by using a 3-(4,5-dimethylthiazol-2-yl)-2,5-diphenyltetrazolium bromide (MTT) assay and dasatinib as the reference drug. Among the tested derivatives, compounds 4, 10, 16, and 19 showed good activity as cytotoxic agents. The most active derivatives were evaluated for their ability to inhibit vascular endothelial growth factor receptor (VEGFR)-2. Compounds $Z$-4-(3-(3,4-dimethoxyphenyl)-3-oxoprop-1-enylamino)- $N$ (5-methyl-1,3,4-thiadiazol-2-yl)-benzenesulfonamide 10 and $Z$-4-(3-(3,4-dimethoxyphenyl)-3-oxoprop-1enylamino)- $N$-( $1 H$-indazol-6-yl)-benzenesulfonamide 19 were more active as VEGFR-2 inhibitors than dasatinib. Molecular docking of the most active derivatives on the active site of VEGFR-2 revealed that compound 19 exhibited favorable and promising results.

Key words growth factor receptor; benzenesulfonamide; 3,4-dimethoxyphenyl; anticancer

Cancer is considered the second common cause of death after cardiovascular diseases, and is expected to become first in the next few years. ${ }^{1,2}$ Chemotherapeutic agents used for therapy have two drawbacks: lack of selectivity as most used drugs act on normal as well as tumor cells, leading to serious side effects ${ }^{3)}$ and refractoriness of tumor cells to traditional anticancer agents. Although the latter may be overcome by using several drug combinations, it will however lead to higher cost and longer treatment durations. $\left.{ }^{4}\right)$ Vascular endothelial growth factor receptor (VEGFR) inhibitors have received great attention over the last decade as novel anticancer agents. ${ }^{5-12}$ ) VEGF is a key angiogenic stimulator secreted by tumor cells to switch on the angiogenic phenotype. ${ }^{13)}$ Moreover, through circulation, angiogenic capillaries stimulate tumor cells to migrate to other sites (metastasis), leading to an increased mortality rate. ${ }^{13)}$ Through binding to a number of receptors, VEGF has become one of the most important regulators of angiogenesis. $^{14,15)}$ This process is mainly facilitated through a specific VEGF receptor, the kinase insert domain-containing receptor (KDR or VEGFR-2), ${ }^{16)}$ which is mostly expressed on vascular endothelial cells, and is up-regulated in angiogenic blood vessels. ${ }^{17)}$ Inhibition of angiogenesis may result in the arrest of tumor growth, a theory that was first proposed by Folkman in $1971,{ }^{18)}$ and has now become a reality, since several VEGFR-2 inhibitors are currently used in clinics as anticancer agents. ${ }^{19-21)}$ Pazopanib I is a VEGFR-2 inhibitor that has been recently approved for the treatment of advanced metastatic renal carcinoma and soft tissue sarcoma, and contains an active benzenesulfonamide moiety in its chemical structure. ${ }^{22-24)}$ Many sulfonamides exhibited favorable anticancer activity through numerous mechanisms including cell cycle arrest in the G1 phase ${ }^{25)}$ and inhibition of carbonic anhydrase (CA), ${ }^{26)}$ histone deacetylases (HDACs), ${ }^{27)}$ methionine aminopeptidases (MetAPs), ${ }^{28)}$ matrix metalloproteinase (MMPs), ${ }^{29)}$ nicotinamide adenine dinucleotide (NADH) oxidase, ${ }^{30)}$ cyclin-dependent kinase (CDK). ${ }^{31)}$ Moreover, by binding to $\beta$-tubulin, they can disrupt the microtubule assembly. ${ }^{32)}$ A different agent, KRN 633 II, is under clinical trials as a VEGFR-2 inhibitor. ${ }^{33)}$ It contains the active moiety 3,4-dimethoxyphenyl. Based on the aforementioned facts and as a continuation of our research on novel anticancer agents, ${ }^{34-40)}$ the current study focuses on the synthesis of novel benzenesulfonamide derivatives bearing the 3,4-dimethoxyphenyl moiety, as VEGFR-2 inhibitors. The most active cytotoxic compounds were evaluated for their VEGFR-2 inhibition activity; moreover, docking of the VEGFR-2 active site was performed to explore the binding mode of the inhibitors. It's worth mentioning that similar work was performed on 4',7-dicholorquinoline moiety with the substitution of different sulfonamides and good cytotoxic activity was observed to several derivatives against four different cell lines. $^{41)}$ 


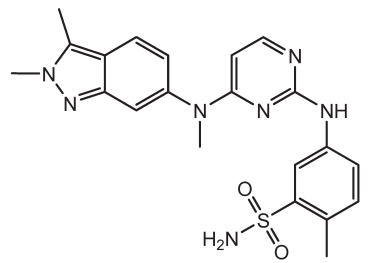

I

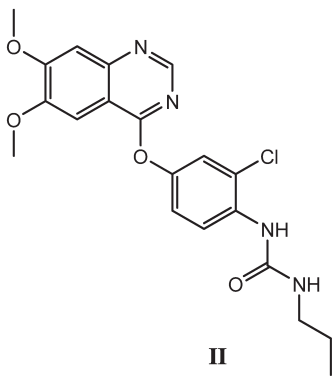

\section{Results and Discussion}

Chemistry The goal of this study was to synthesize a novel series of benzenesulfonamides with a biologically active 3,4-dimethoxyphenyl moiety and to assess their anticancer activity. Thus, reaction of 3,4-dimethoxyacetophenone 1 with dimethylformamide-dimethylacetal in dry xylene yielded the starting material E-1-(3,4-dimethoxyphenyl)-3(dimethylamino)prop-2-en-1-one 2. Enaminone 2 was allocated an E-configuration based on its ${ }^{1} \mathrm{H}-\mathrm{NMR}$ spectrum, which indicated that the coupling constant of the doublet signals for olefinic protons was equivalent to $12.3 \mathrm{~Hz}$ and associated to $E$-isomers. Reaction of enaminone 2 with sulfa drugs in absolute ethanol and glacial acetic acid $(2: 1)$ resulted in benzenesulfonamide derivatives 3-19 at a reasonable yield (Chart 1). The structures of the obtained products were established using microanalysis, IR, ${ }^{1} \mathrm{H}-\mathrm{NMR}$, and ${ }^{13} \mathrm{C}-\mathrm{NMR}$ spectral data. The ${ }^{1} \mathrm{H}-\mathrm{NMR}$ spectra for compounds 3-19 revealed that these structures were in the $Z$-, and not in the $E$-form, while the coupling constant of the doublet signals for olefinic protons was $8.5-8.6 \mathrm{~Hz}$. Moreover, the $Z$-form is stabilized by intramolecular hydrogen bonds (Chart 1). Similar reaction of 2-enaminone derivative in $E$-configuration with several primary amines revealed $Z$-configuration products were reported in literature. ${ }^{42)}$ The ${ }^{1} \mathrm{H}-\mathrm{NMR}$ spectra of $\mathbf{3}$ shows a singlet at 10.03 ppm for $\mathrm{SO}_{2} \mathrm{NH}_{2}$ that was exchangeable with $\mathrm{D}_{2} \mathrm{O}$. The ${ }^{1} \mathrm{H}-\mathrm{NMR}$ spectra of $\mathbf{4}$ revealed a singlet at $1.92 \mathrm{ppm}$ for the $\mathrm{COCH}_{3}$ group whereas that of $\mathbf{6}$ showed a singlet at $2.30 \mathrm{ppm}$ for the $\mathrm{CH}_{3}$ group and a singlet at $6.25 \mathrm{ppm}$ for the isoxazole $\mathrm{CH}$. The ${ }^{1} \mathrm{H}-\mathrm{NMR}$ spectra of 7 revealed two singlets at 2.07 and $2.09 \mathrm{ppm}$ for the $2 \mathrm{CH}_{3}$ groups whereas that of $\mathbf{8}$ showed a doublet signal at $5.90 \mathrm{ppm}$ for the $2 \mathrm{CH}$ groups of pyrazole. The ${ }^{1} \mathrm{H}-\mathrm{NMR}$ spectra of $\mathbf{1 0}$ exhibited a singlet at $2.44 \mathrm{ppm}$ for the $\mathrm{CH}_{3}$ group whereas that of $\mathbf{1 3}$ presented a singlet at $2.30 \mathrm{ppm}$ for the $\mathrm{CH}_{3}$ group and that of $\mathbf{1 4}$ revealed a singlet at $2.24 \mathrm{ppm}$ for $2 \mathrm{CH}_{3}$ groups and a singlet at $5.96 \mathrm{ppm}$ for the pyrimidine $\mathrm{CH}$. The ${ }^{1} \mathrm{H}-\mathrm{NMR}$ spectra of $\mathbf{1 5}$ displayed a singlet at $2.51 \mathrm{ppm}$ for $2 \mathrm{CH}_{3}$ groups whereas that of $\mathbf{1 7}$ indicated the presence of two singlets at 3.78 and $3.79 \mathrm{ppm}$ for $2 \mathrm{OCH}_{3}$ groups, and a singlet at $5.96 \mathrm{ppm}$ for the pyrimidine $\mathrm{CH}$. The ${ }^{1} \mathrm{H}-\mathrm{NMR}$ spectra of $\mathbf{1 8}$ showed two singlets at 3.70 and $3.90 \mathrm{ppm}$ for $2 \mathrm{OCH}_{3}$ groups, and a singlet at $8.12 \mathrm{ppm}$ for the pyrimidine $\mathrm{CH}$ whereas that of $\mathbf{1 9}$ showed a singlet signal at $8.13 \mathrm{ppm}$ for the pyrazole $\mathrm{CH}$.

In Vitro Anticancer Activity The synthesized compounds were evaluated for their cytotoxic activity against the human cervical cancer (HeLa), human hepatocellular carcinoma (HepG2), human medulloblastoma (Daoy), and human colon cancer (HT-29) cells by using an 3-(4,5-dimethylthiazol2-yl)-2,5-diphenyltetrazolium bromide (MTT) assay, and da-
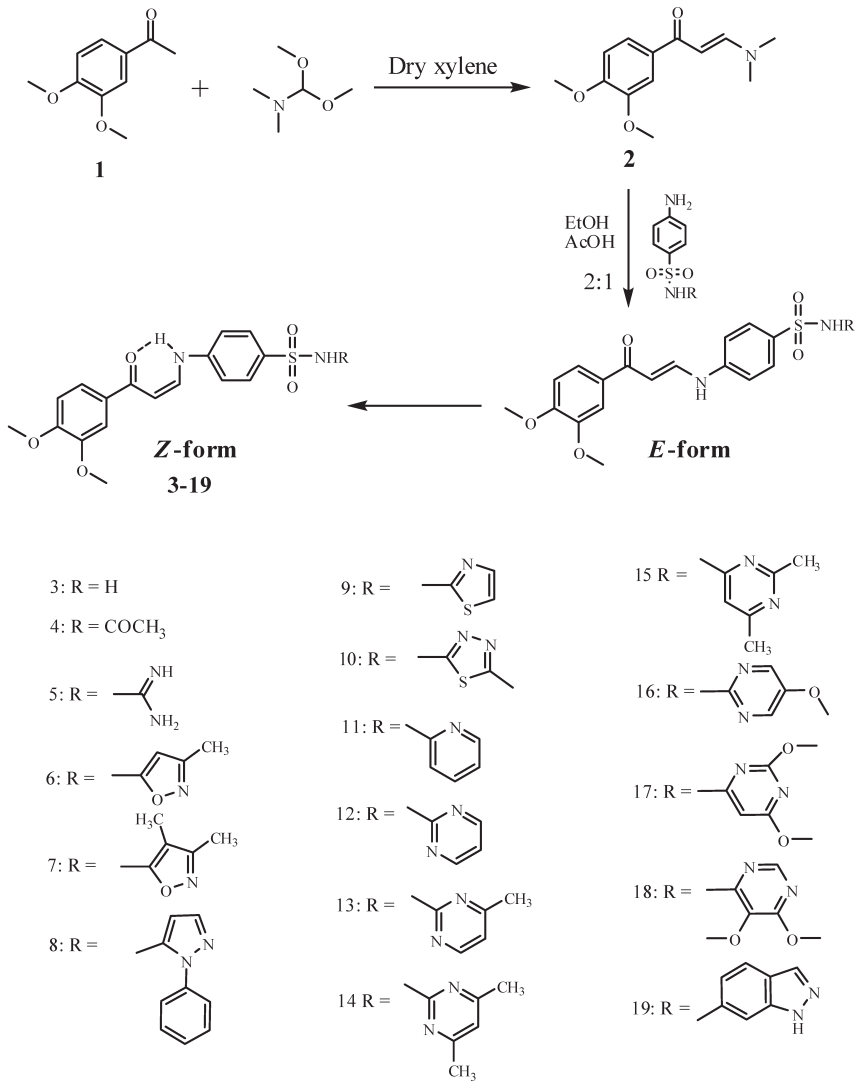

Chart 1. Synthetic Pathways for Compounds 3-19

satinib as the reference drug. Percentages of growth inhibition for all compounds as well as the $\mathrm{IC}_{50}$ for the most active compounds are listed in Table 1. Compound $\mathbf{1}$ inhibited HepG2 cell growth by $58.36 \%$ with an $\mathrm{IC}_{50}$ of $14.17 \mu \mathrm{g} / \mathrm{mL}$. However, the percentage of inhibition for the remaining cell lines was below 50. In contrast, compounds 4,10 and 19 showed over $50 \%$ growth inhibition of HeLa, HepG2, and Daoy cells, but below $50 \%$ for HT-29 cells. Compound $\mathbf{1 6}$ was the only compound that showed over $50 \%$ growth inhibition of all the cancer cell lines tested. Compound $\mathbf{4}$ was the most active against $\mathrm{HeLa}$ cells with a remarkable $\mathrm{IC}_{50}$ of $1.78 \mu \mathrm{g} / \mathrm{mL}$, an order of magnitude more potent than dasatinib $\left(\mathrm{IC}_{50}: 16.22 \mu \mathrm{g} /\right.$ $\mathrm{mL}$ ). Compound 19 was the most active against HepG2 cells, with an $\mathrm{IC}_{50}$ of $1.6 \mu \mathrm{g} / \mathrm{mL}$, and was more potent than dasatinib $\left(\mathrm{IC}_{50}: 6.91 \mu \mathrm{g} / \mathrm{mL}\right)$. The same compound was also the most active against Daoy cells with an $\mathrm{IC}_{50}$ of $5.58 \mu \mathrm{g} / \mathrm{mL}$, almost twice as potent as dasatinib $\left(\mathrm{IC}_{50}: 9.2 \mu \mathrm{g} / \mathrm{mL}\right)$.

Structure-Activity Relationship

It appeared obvious that the substitution of sulfonamide derivatives used in the synthesis of compounds 3-19 has a remarkable effect on their cytotoxic activity. The acetyl derivative 4 showed favorable activity against HepG2 cells, whereas both the unsubstituted sulfonamide derivative $\mathbf{3}$ and the guanido derivative $\mathbf{5}$ exhibited low activity. Among the various heterocyclic sulfonamide derivatives 6-19, the methyl thidiazole derivative $\mathbf{1 0}$, the 5-methoxy pyrimidine derivative 16, and the benzopyrazole derivative 19 displayed an interesting activity against HepG2 and Daoy cells.

VEGFR-2 Inhibition Activity The most active compounds $(4,10,16,19)$ were evaluated for their in vitro VEGFR-2 inhibitory activity in the HeLa cell line, using da- 
Table 1. In Vitro Cytotoxicity of New Compounds against Cancer Cell Lines

\begin{tabular}{|c|c|c|c|c|c|c|c|c|}
\hline \multirow{2}{*}{ Compd. } & \multicolumn{2}{|c|}{ Hela } & \multicolumn{2}{|c|}{ HepG-2 } & \multicolumn{2}{|c|}{ Daoy } & \multicolumn{2}{|c|}{ HT-29 } \\
\hline & $\%$ Inhibition* & $\mathrm{IC}_{50} * *$ & \% Inhibition* & $\mathrm{IC}_{50} * *$ & $\%$ Inhibition* & $\mathrm{IC}_{50} * *$ & $\%$ Inhibition* & $\mathrm{IC}_{50} * *$ \\
\hline 1 & 45.9 & $\mathrm{nt}^{\#}$ & 58.36 & $14.17 \pm 0.83$ & 41.37 & $\mathrm{nt}^{\#}$ & 34.83 & $\mathrm{nt}^{\#}$ \\
\hline 2 & 32.53 & $\mathrm{nt}^{\#}$ & 33.76 & $\mathrm{nt}^{\#}$ & 29.78 & $\mathrm{nt}^{\#}$ & 35.82 & $\mathrm{nt}^{\#}$ \\
\hline 3 & 30.91 & $\mathrm{nt}^{\#}$ & 34.36 & $\mathrm{nt}^{\#}$ & 3.34 & $\mathrm{nt}^{\#}$ & 0 & $\mathrm{nt}^{\#}$ \\
\hline 4 & 58.26 & $1.78 \pm 0.08$ & 63.41 & $2.63 \pm 0.13$ & 55.3 & $7.74 \pm 0.26$ & 46.05 & $\mathrm{nt}^{\#}$ \\
\hline 5 & 37.48 & $\mathrm{nt}^{\#}$ & 31.32 & $\mathrm{nt}^{\#}$ & 23.67 & $\mathrm{nt}^{\#}$ & 28.2 & $\mathrm{nt}^{\#}$ \\
\hline 6 & 9.36 & $\mathrm{nt}^{\#}$ & 5.91 & $\mathrm{nt}^{\#}$ & 41.49 & $\mathrm{nt}^{\#}$ & 0 & $\mathrm{nt}^{\#}$ \\
\hline 7 & 36.72 & $\mathrm{nt}^{\#}$ & 36.04 & $\mathrm{nt}^{\#}$ & 30.3 & $\mathrm{nt}^{\#}$ & 0 & $\mathrm{nt}^{\#}$ \\
\hline 8 & 46.1 & $\mathrm{nt}^{\#}$ & 46.69 & $\mathrm{nt}^{\#}$ & 35.59 & $\mathrm{nt}^{\#}$ & 36.39 & $\mathrm{nt}^{\#}$ \\
\hline 9 & 29.32 & $\mathrm{nt}^{\#}$ & 30.21 & $\mathrm{nt}^{\#}$ & 36.82 & $\mathrm{nt}^{\#}$ & 19.44 & $\mathrm{nt}^{\#}$ \\
\hline 10 & 57.51 & $20.45 \pm 0.15$ & 56.5 & $20.78 \pm 0.68$ & 51.32 & $19.9 \pm 1.6$ & 33.7 & $\mathrm{nt}^{\#}$ \\
\hline 11 & 26.72 & $n t^{\#}$ & 21.65 & $\mathrm{nt}^{\#}$ & 24.86 & $\mathrm{nt}^{\#}$ & 0 & $\mathrm{nt}^{\#}$ \\
\hline 12 & 17.12 & $\mathrm{nt}^{\#}$ & 33.03 & $\mathrm{nt}^{\#}$ & 17.12 & $\mathrm{nt}^{\#}$ & 3.7 & $\mathrm{nt}^{\#}$ \\
\hline 13 & 22.6 & $\mathrm{nt}^{\#}$ & 5.5 & $\mathrm{nt}^{\#}$ & 22.6 & $\mathrm{nt}^{\#}$ & 0 & $\mathrm{nt}^{\#}$ \\
\hline 14 & 6.04 & $\mathrm{nt}^{\#}$ & 0 & $\mathrm{nt}^{\#}$ & 6.04 & $\mathrm{nt}^{\#}$ & 0 & $\mathrm{nt}^{\#}$ \\
\hline 15 & 44.07 & $\mathrm{nt}^{\#}$ & 44.66 & $\mathrm{nt}^{\#}$ & 44.7 & $\mathrm{nt}^{\#}$ & 3.65 & $\mathrm{nt}^{\#}$ \\
\hline 16 & 57.27 & $22.5 \pm 0.9$ & 61 & $20.74 \pm 0.48$ & 53.02 & $18.44 \pm 0.84$ & 52.03 & $24.46 \pm 1.46$ \\
\hline 17 & 39.24 & $\mathrm{nt}^{\#}$ & 41.17 & $\mathrm{nt}^{\#}$ & 35.1 & $\mathrm{nt}^{\#}$ & 15.26 & $\mathrm{nt}^{\#}$ \\
\hline 18 & 24.79 & $\mathrm{nt}^{\#}$ & 24.45 & $\mathrm{nt}^{\#}$ & 17.59 & $\mathrm{nt}^{\#}$ & 0 & $\mathrm{nt}^{\#}$ \\
\hline 19 & 60.6 & $2.69 \pm 0.19$ & 68.49 & $1.6 \pm 0.2$ & 56.12 & $5.58 \pm 0.42$ & 39.21 & $\mathrm{nt}^{\#}$ \\
\hline Dasatinib & 56.4 & $16.22 \pm 0.78$ & 73.98 & $6.91 \pm 0.31$ & 90.36 & $9.2 \pm 0.2$ & 39.48 & $25.56 \pm 1.44$ \\
\hline
\end{tabular}

* Percent of growth inhibition of cells at a concentration of $25 \mu \mathrm{g} / \mathrm{mL}$, relative to control. ** Dose-response curves were used to calculate $\mathrm{IC}_{50}$ values $(\mu \mathrm{g} / \mathrm{mL})$, expressed as the mean \pm S.D. ${ }^{\#}$ nt: Not tested.

Table 2. In Vitro Inhibition of VEGFR-2 for the Most Active Compounds

\begin{tabular}{ccccc}
\hline \hline Compounds & $M_{\mathrm{w}}(\mathrm{g} / \mathrm{mol})$ & $\mathrm{IC}_{50}$ values $(\mu \mathrm{g} / \mathrm{mL})$ & VEGFR residual (conc. pg/mL) & VEGFR \% inhibition \\
\hline $\mathbf{4}$ & 404 & 1.78 & 686.491 & 72.8 \\
$\mathbf{1 0}$ & 460 & 20.45 & 740.914 & 80.6 \\
$\mathbf{1 6}$ & 470 & 22.5 & 775.862 & 74.7 \\
$\mathbf{1 9}$ & 478 & 2.69 & 590.541 & 84.2 \\
Dasatinib & 488 & 0.8 & 978.443 & 79.5 \\
\hline
\end{tabular}

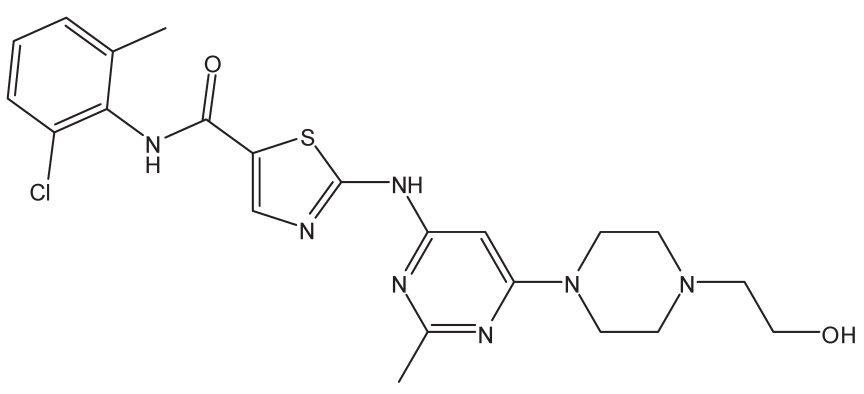

Dasatinib

satinib as the reference drug. All four compounds showed a good inhibition (72.8-84.2\% of control). Compounds 10 and 19 were more active than the reference drug (79.5\%) with an inhibition of 80.6 and $84.2 \%$, respectively. VEGFR-2 residual concentrations as well as VEGFR-2 inhibition percentages for the tested compounds are listed in Table 2. These interesting results urge to synthesize and evaluate VEGFR-2 inhibition activity of more derivatives using other sulfonamide moieties in the future.

Molecular Docking In order to explore the potential binding mode of the most active compounds to VEGFR-2, compounds 4, 10, 16, and 19 were docked on the receptor active site. The protein databank file (PDB ID) 4AGD was selected for this purpose. The file contains data on VEGFR-2 co-crystallized with sunitinib, a type I VEGFR-2 inhibitor. Type I inhibitors bind only to the ATP binding site of kinases with the hinge region with no interactions with allosteric pocket. All docking procedures were achieved by MOE (Molecular Operating Environment) software 10.2008 provided by the chemical computing group, Canada. Docking on the VEGFR-2 active site was performed for the sulfonamide derivatives $4,10,16$, and 19 . The docking protocol was verified by re-docking the co-crystallized ligand near the active site with an energy score (S) of $-22.9143 \mathrm{kcal} / \mathrm{mol}$ and a root mean standard deviation (RMSD) of 1.5923. The cocrystalized inhibitor interacts with the active site of VEGFR-2 via two hydrogen bonds: the first, between Glu917 and the nitrogen of the pyrrole moiety, and the second, between Cys919 and the carbonyl group (Fig. 1).

The four compounds, $4,10,16$, and 19 were fit in the active site of VEGFR-2 with energy scores ranging between -11.9827 to $-19.6112 \mathrm{kcal} / \mathrm{mol}$. Molecular docking for pazopanib, KNR 633 and dasatinib was also performed. The energy scores of the docked compounds and the amino acid interactions are listed in Table 3.

The optimal docking energy score among the synthesized compounds was displayed by compound 19 with a value of $-19.6112 \mathrm{kcal} / \mathrm{mol}$ supporting its excellent activity as a 
Table 3. Docking Score (S) and Amino Acid Interactions for the Most Active Compounds on the VEGFR-2 Active Site

\begin{tabular}{|c|c|c|c|c|c|}
\hline Compounds & (S) $(\mathrm{kcal} / \mathrm{mol})$ & Amino acid & Interacting group & Type of interaction & H-Bond length $(\AA)$ \\
\hline \multirow[t]{2}{*}{4} & -13.5853 & Lys838 & $\mathrm{C}=\mathrm{O}$ & $\mathrm{H}$ bond (acceptor) & 2.49 \\
\hline & & Cys919 & $\mathrm{C}=\mathrm{O}$ & $\mathrm{H}$ bond (acceptor) & 2.70 \\
\hline 10 & -11.9827 & Cys919 & $\mathrm{SO}_{2}$ & H bond (acceptor) & 2.99 \\
\hline 16 & -13.9947 & Cys919 & $\mathrm{OCH}_{3}$ & H bond (acceptor) & 2.99 \\
\hline \multirow[t]{3}{*}{19} & -19.6112 & Lys838 & $\mathrm{C}=\mathrm{O}$ & $\mathrm{H}$ bond (acceptor) & 2.48 \\
\hline & & Glu917 & NH pyrazole & $\mathrm{H}$ bond (donor) & 2.02 \\
\hline & & Cys919 & $\mathrm{SO}_{2}$ & $\mathrm{H}$ bond (acceptor) & 3.03 \\
\hline Pazopanib & -20.6243 & Cys919 & N pyrazole & H bond (acceptor) & 3.18 \\
\hline KRN 633 & -17.8653 & Cys919 & $\mathrm{N}$ pyrimidin & H bond (acceptor) & 2.89 \\
\hline Dasatinib & -8.2171 & Cys919 & $\mathrm{C}=\mathrm{O}$ & $\mathrm{H}$ bond (acceptor) & 3.05 \\
\hline \multirow[t]{2}{*}{ Sunitinib } & -22.9143 & Glu917 & $\mathrm{N}$ pyrrole & $\mathrm{H}$ bond (donor) & 2.93 \\
\hline & & Cys919 & $\mathrm{C}=\mathrm{O}$ & $\mathrm{H}$ bond (acceptor) & 2.92 \\
\hline
\end{tabular}

(8ia)

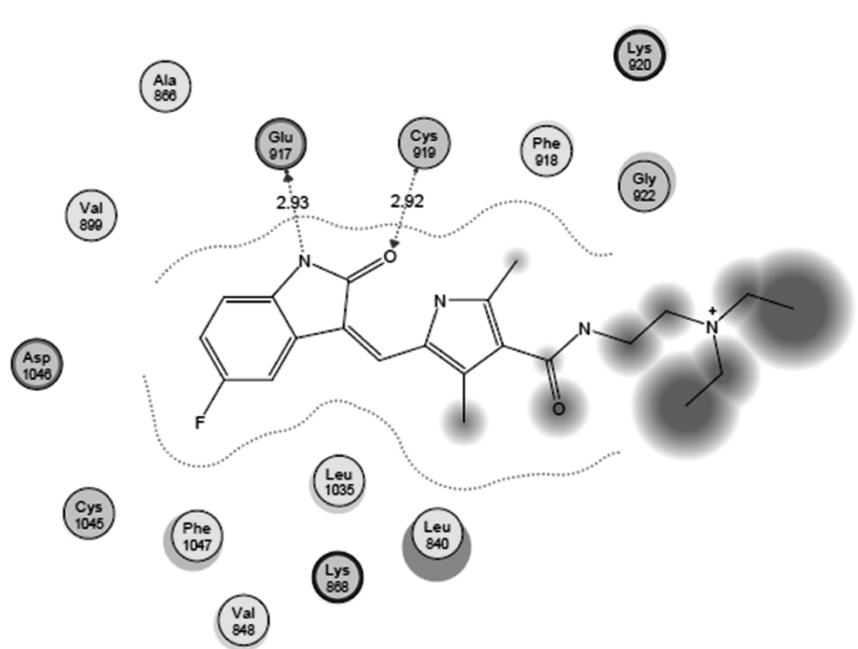

Fig. 1. Co-crystallized Ligand on the Active Site of VEGFR-2

VEGFR-2 inhibitor (84.2\% growth inhibition rate). Compound 19 interacted with the same amino acids (Glu917 and Cys919) in a comparable manner to the co-crystallized ligand did, and had an additional hydrogen bond with Lys838. The amino acid interactions exhibited by compound $\mathbf{1 9}$ are illustrated in Figs. 2 and 3. Amino acid interactions of pazopanib, KRN 633 and dasatinib are illustrated in Figs. 4-6, respectively.

\section{Experimental}

Chemistry Elemental analyses were performed using a model $2400 \mathrm{CHNS} / \mathrm{O}$ analyzer (PerkinElmer, Inc., U.S.A.). All the values were within $\pm 0.4 \%$ of the theoretical values; melting points ( $\mathrm{mp}$; uncorrected) were determined using an open capillary on a Gallenkamp melting point apparatus (Sanyo Gallenkamp, U.K.). Precoated silica gel plates (Kieselgel $0.25 \mathrm{~mm}, 60 \mathrm{~F} 254$, Merck, Germany) were used for thin layer chromatography. A developing solvent system of chloroform-methanol $(8: 2)$ was used, and the spots were detected by ultraviolet light. IR spectra ( $\mathrm{KBr}$ disc) were recorded using a Fourier transform infrared (FT-IR) spectrophotometer (PerkinElmer, Inc., U.S.A.). ${ }^{1} \mathrm{H}-\mathrm{NMR}$ spectra were scanned on a NMR spectrophotometer (Bruker AXS Inc., Switzerland), op- val

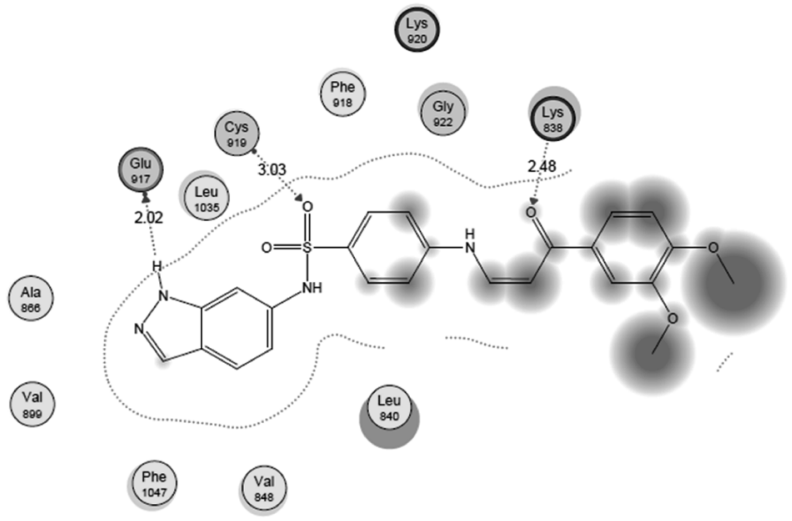

Fig. 2. 2D Image of Compound 19 Docked onto the VEGFR-2 Active Site

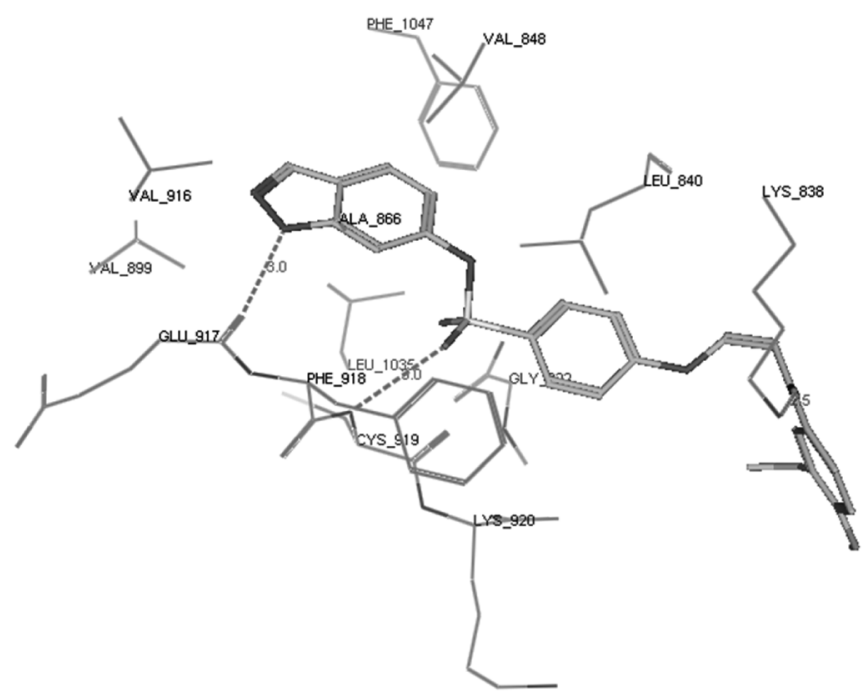

Fig. 3. 3D Image of Compound 19 Docked to the VEGFR-2 Active Site

erating at $500 \mathrm{MHz}$ for ${ }^{1} \mathrm{H}$, and $125.76 \mathrm{MHz}$ for ${ }^{13} \mathrm{C}$. Chemical shifts are expressed as $\delta$-values (ppm) relative to tetramethylsilane (TMS) as an internal standard, and dimethyl sulfoxide (DMSO)- $d_{6}$ as a solvent. All reagents used were of analytical grade. The starting material 3,4-dimethoxyacetophenone 1 was purchased from Sigma-Aldrich (St. Louis, MO, U.S.A.), and was directly used for the preparation of the target com- 


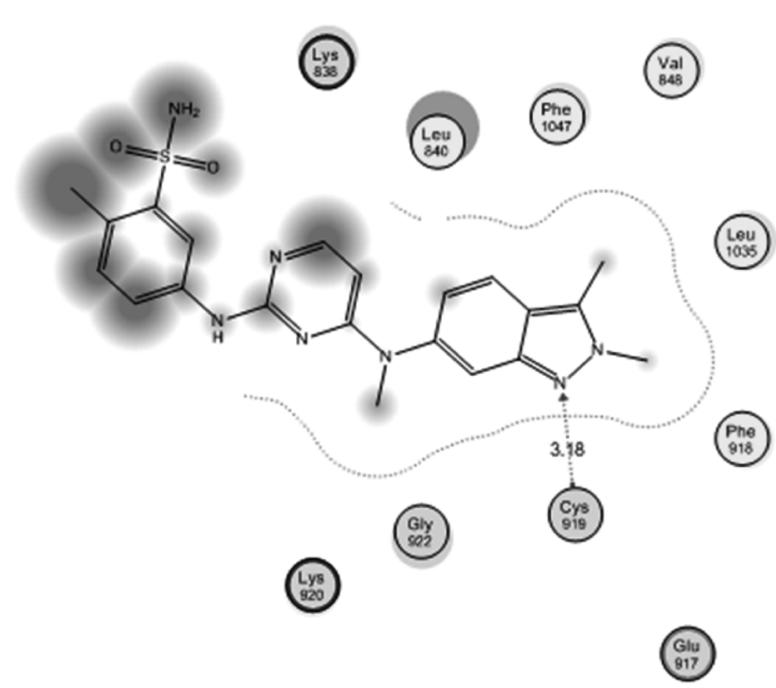

Fig. 4. 2D Image of Pazopanib Docked onto the VEGFR-2 Active Site

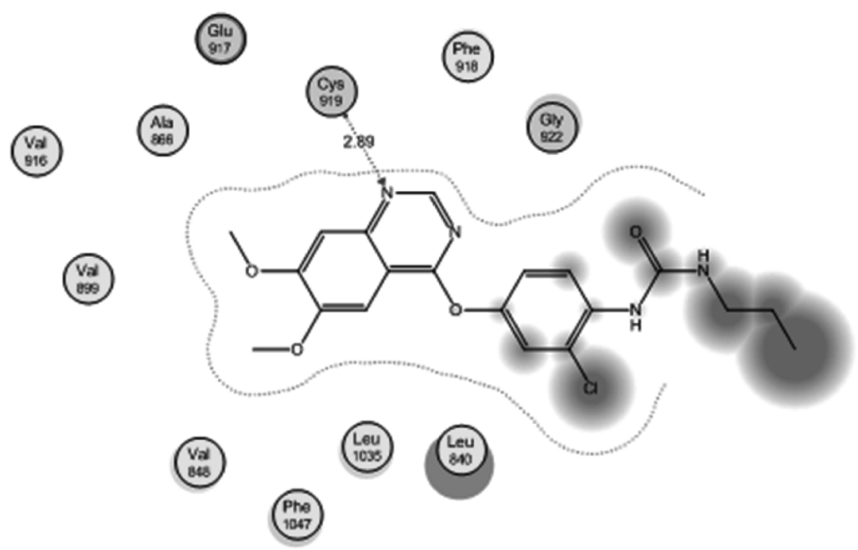

Fig. 5. 2D Image of KRN 633 Docked onto the VEGFR-2 Active Site

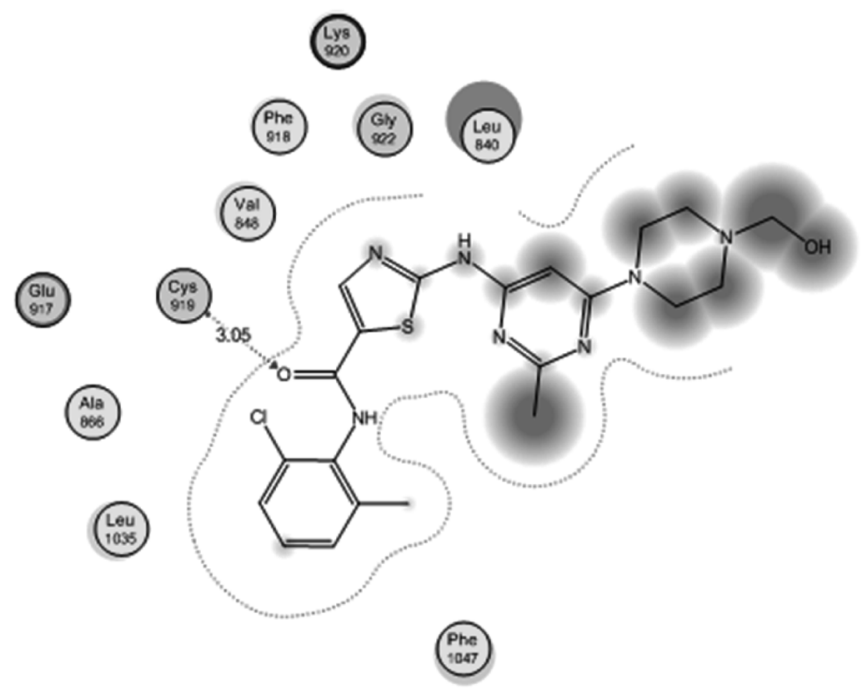

Fig. 6. 2D Image of Dasatinib Docked onto the VEGFR-2 Active Site

pounds.

Z-1-(3,4-Dimethxoyphenyl)-3-(dimethylamino)prop-2-en-1one (2)

A solution of 3,4-dimethoxyacetophenone $(1.8 \mathrm{~g}, 0.01 \mathrm{~mol})$ in dimethylformamide-dimethylacetal $(1.19 \mathrm{~g}, 0.01 \mathrm{~mol})$ was refluxed in dry xylene $(20 \mathrm{~mL})$ for $24 \mathrm{~h}$. The obtained solid was collected by filtration, while still hot, and recrystallized from dioxane to yield compound 2 .

Yield, 66\%; mp $123.7^{\circ} \mathrm{C}$. IR: 3080 (arom.), 2997, 2931, 2839 (aliph.), 1635 (CO). ${ }^{1} \mathrm{H}-\mathrm{NMR}: 3.11$ (s, 6H, 2NCH${ }_{3}$ ), 3.80 (s, 6H, $\left.2 \mathrm{OCH}_{3}\right), 5.82,6.98(2 \mathrm{~d}, 2 \mathrm{H}, \mathrm{CH}=\mathrm{CH}, J=12.3 \mathrm{~Hz}), 7.47-7.69$ (m, 3H, Ar-H). ${ }^{13} \mathrm{C}-\mathrm{NMR}: 44.8(2), 56.0(2), 91.1,110.8,111.1$, 121.2, 133.4, 148.7, 151.7, 154.1, 185.3. MS $m / z(\%): 235\left(\mathrm{M}^{+}\right)$ (12.73), 136 (100). Anal. Calcd for $\mathrm{C}_{13} \mathrm{H}_{17} \mathrm{NO}_{3}$ (235): C, 66.36; H, 7.28; N, 5.95. Found: C, 66.07; H, 7.54; N, 6.23.

Synthesis of Benzenesulfonamide Derivatives (3-19)

General Procedure

A mixture of $2(2.35 \mathrm{~g}, 0.01 \mathrm{~mol})$ and sulfa drugs $(0.012 \mathrm{~mol})$ in absolute ethanol $(10 \mathrm{~mL})$ and glacial acetic acid $(5 \mathrm{~mL})$ was refluxed for $22 \mathrm{~h}$, and subsequently left to cool. The solid product formed was collected by filtration and recrystallized from acetic acid to give compounds 3-19.

Z-4-(3-(3,4-Dimethoxyphenyl)-3-oxoprop-1-enylamino)benzenesulfonamide (3)

Yield, 89\%; mp $276.4^{\circ} \mathrm{C}$. IR: 3329, $3236\left(\mathrm{NH}_{2}, \mathrm{NH}\right), 3005$ (arom.), 2974, 2939, 2839 (aliph.), 1635 (CO), 1375, $1188\left(\mathrm{SO}_{2}\right)$. ${ }^{1} \mathrm{H}-\mathrm{NMR}: 3.83\left(\mathrm{~s}, 6 \mathrm{H}, 2 \mathrm{OCH}_{3}\right), 6.23,6.60(2 \mathrm{~d}, 2 \mathrm{H}, \mathrm{CH}=\mathrm{CH}$, $J=8.5 \mathrm{~Hz}), 7.03-8.15(\mathrm{~m}, 7 \mathrm{H}, \mathrm{Ar}-\mathrm{H}), 10.30$ (s, 2H, $\mathrm{SO}_{2} \mathrm{NH}_{2}$ ), 12.04 (s, 1H, NH). ${ }^{13} \mathrm{C}-\mathrm{NMR}: 55.9,56.1,95.2,110.5,111.3$, $111.4,115.2,121.7,127.9,128.1,131.5,132.2,142.8,144.5$, 149.0, 152.7, 189.6. MS m/z (\%): $362\left(\mathrm{M}^{+}\right)$(6.48), 155 (100). Anal. Calcd for $\mathrm{C}_{17} \mathrm{H}_{18} \mathrm{~N}_{2} \mathrm{O}_{5} \mathrm{~S}$ (362): C, 56.34; H, 5.01; N, 7.73. Found: C, 56.62; H, 5.29; N, 7.46.

$Z$-N-(4-(3-(3,4-Dimethoxyphenyl)-3-oxoprop-1-enylamino)phenylsulfonyl)acetamide (4)

Yield, 87\%; mp $215.5^{\circ} \mathrm{C}$. IR: 3329, $3236(\mathrm{NH}), 3078$ (arom.), 2974, 2939, 2841 (aliph.), 1718, 1635 (2CO), 1373, $1155\left(\mathrm{SO}_{2}\right)$. ${ }^{1} \mathrm{H}-\mathrm{NMR}: 1.92\left[\mathrm{~s}, 3 \mathrm{H}, \mathrm{COCH}_{3}\right], 3.83\left[\mathrm{~s}, 6 \mathrm{H}, 2 \mathrm{OCH}_{3}\right], 6.22$, $6.61[2 \mathrm{~d}, 2 \mathrm{H}, \mathrm{CH}=\mathrm{CH}, J=8.6 \mathrm{~Hz}] .7 .02-8.17$ [m, 7H, Ar-H], $10.40\left[\mathrm{~s}, 1 \mathrm{H}, \mathrm{SO}_{2} \mathrm{NH}\right], 12.03[\mathrm{~s}, 1 \mathrm{H}, \mathrm{NH}] .{ }^{13} \mathrm{C}-\mathrm{NMR}: 23.6$, 55.9, 56.0, 95.2, 110.5, 111.3, 115.1, 115.2, 121.7, 122.0, 130.1(2), 131.4, 143.8, 145.1, 152.7, 152.8, 169.2, 189.6. MS m/z (\%): 404 $\left(\mathrm{M}^{+}\right)$(2.65), 93 (100). Anal. Calcd for $\mathrm{C}_{19} \mathrm{H}_{20} \mathrm{~N}_{2} \mathrm{O}_{6} \mathrm{~S}$ (404): C, 56.42; H, 4.98; N, 6.93. Found: C, 56.16; H, 5.35; N, 7.26.

$\mathrm{Z}-\mathrm{N}$-Carbamimidoyl-4-(3-(3,4-dimethoxy phenyl)-3oxoprop-1-enylamino)benzenesulfonamide (5)

Yield, 91\%; mp $260.1^{\circ} \mathrm{C}$. IR: 3435, 3336, $3228\left(\mathrm{NH}_{2}, \mathrm{NH}\right)$, 3100 (arom.), 2976, 2939, 2843 (aliph.), 1635 (CO), 1593 (CN), 1375, $1166\left(\mathrm{SO}_{2}\right) .{ }^{1} \mathrm{H}-\mathrm{NMR}: 3.83\left(\mathrm{~s}, 6 \mathrm{H}, 2 \mathrm{OCH}_{3}\right), 6.20,6.57$ $(2 \mathrm{~d}, 2 \mathrm{H}, \mathrm{CH}=\mathrm{CH}, J=8.6 \mathrm{~Hz}), 7.03-7.73\left(\mathrm{~m}, 9 \mathrm{H}, \mathrm{Ar}-\mathrm{H}+\mathrm{NH}_{2}\right)$, $8.13\left(\mathrm{~s}, 1 \mathrm{H}, \mathrm{SO}_{2} \mathrm{NH}\right), 10.15(\mathrm{~s}, 1 \mathrm{H}, \mathrm{NH}), 12.03$ (s, 1H, NH, imino). ${ }^{13} \mathrm{C}-\mathrm{NMR}: 55.9,56.1,94.9,110.4,111.3,115.1,115.2$, 121.7, 127.8, 131.6(2), 133.3, 142.9, 144.5, 152.5, 158.4, 158.5, 189.5. MS m/z (\%): $404\left(\mathrm{M}^{+}\right)$(17.22), 77 (100). Anal. Calcd for $\mathrm{C}_{18} \mathrm{H}_{20} \mathrm{~N}_{4} \mathrm{O}_{5} \mathrm{~S}$ (404): C, 53.45; H, 4.98; N, 13.85. Found: C, 53.16; H, 5.31; N, 13.56 .

Z-4-(3-(3,4-Dimethoxyphenyl)-3-oxoprop-1-enylamino)- $N$ (3-methylisoxaz-ol-5-yl)benzenesulfonamide (6)

Yield, 88\%; mp $160.3^{\circ} \mathrm{C}$. IR: 3161(NH), 3066 (arom.), 2978, 2889, 2839 (aliph.), 1637 (CO), 1589 (CN), 1375, $1161\left(\mathrm{SO}_{2}\right)$. ${ }^{1} \mathrm{H}-\mathrm{NMR}: 2.30\left(\mathrm{~s}, 3 \mathrm{H}, \mathrm{CH}_{3}\right), 3.83\left(\mathrm{~s}, 6 \mathrm{H}, 2 \mathrm{OCH}_{3}\right), 6.14,6.61$ $(2 \mathrm{~d}, 2 \mathrm{H}, \mathrm{CH}=\mathrm{CH}, J=8.5 \mathrm{~Hz}), 6.25(\mathrm{~s}, 1 \mathrm{H}, \mathrm{CH}$ isoxazole), 7.03-8.15 (m, 7H, Ar-H), 10.40 (s, 1H, $\left.\mathrm{SO}_{2} \mathrm{NH}\right), 12.00$ (s, $1 \mathrm{H}, \mathrm{NH}) .{ }^{13} \mathrm{C}-\mathrm{NMR}: 12.4,56.1(2), 95.8,100.7,110.5,111.3$, $113.0(2), 121.8,122.0,129.2,131.8(2), 142.4,145.9,149.1$, 
152.8, 158.0, 170.8, 189.7. MS $m / z(\%): 443\left(\mathrm{M}^{+}\right)$(37.18), 96 (100). Anal. Calcd for $\mathrm{C}_{21} \mathrm{H}_{21} \mathrm{~N}_{3} \mathrm{O}_{6} \mathrm{~S}$ (443): C, 56.87; H, 4.77; N, 9.48. Found: C, 56.52; H, 4.40; N, 9.11.

$\mathrm{Z}$-4-(3-(3,4-Dimethoxyphenyl)-3-oxoprop-1-enylamino)- $\mathrm{N}$ (3,4-dimethylisoxazol-5-yl)benzenesulfonamide (7)

Yield, 86\%; mp $238.2^{\circ} \mathrm{C}$. IR: $3334(\mathrm{NH}), 3089$ (arom.), 2960, 2835 (aliph.), 1635 (CO), 1589 (CN), 1346, $1159\left(\mathrm{SO}_{2}\right)$. ${ }^{1} \mathrm{H}-\mathrm{NMR}: 2.07,2.09\left(2 \mathrm{~s}, 3 \mathrm{H}, 2 \mathrm{CH}_{3}\right), 3.83\left(\mathrm{~s}, 6 \mathrm{H}, 2 \mathrm{OCH}_{3}\right)$, $6.26,6.63(2 \mathrm{~d}, 2 \mathrm{H}, \mathrm{CH}=\mathrm{CH}, J=8.5 \mathrm{~Hz}), 7.03-8.16(\mathrm{~m}, 7 \mathrm{H}$, Ar-H), 10.42 (s, 1H, SO $\left.\mathrm{SH}_{2}\right), 12.03$ (s, 1H, NH). ${ }^{13} \mathrm{C}-\mathrm{NMR}$ : 6.3, 10.7, 56.10, 56.12, 95.8, 100.7, 105.5, 110.6, 111.4(2), 121.8, $122.0,129.1,129.2(2), 143.8,145.9,149.1,152.8,156.1,161.9$, 189.7. MS m/z (\%): $457\left(\mathrm{M}^{+}\right)$(31.52), 135 (100). Anal. Calcd for $\mathrm{C}_{22} \mathrm{H}_{23} \mathrm{~N}_{3} \mathrm{O}_{6} \mathrm{~S}$ (457): C, 57.76; H, 5.07; N, 9.18. Found: C, 57.98; $\mathrm{H}, 5.36 ; \mathrm{N}, 9.39$.

Z-4-(3-(3,4-Dimethoxyphenyl)-3-oxoprop-1-enylamino)- $N$ (1-phenyl-1H-pyrazol-5-yl)-benzenesulfonamide (8)

Yield, 80\%; mp $236.1^{\circ} \mathrm{C}$. IR: 3415(NH), 3049 (arom.), 2966, 2835, 2802 (aliph.), 1653 (CO), $1570(\mathrm{CN}), 1338,1159\left(\mathrm{SO}_{2}\right)$. ${ }^{1} \mathrm{H}-\mathrm{NMR}: 3.84\left(\mathrm{~s}, 6 \mathrm{H}, 2 \mathrm{OCH}_{3}\right), 5.90$ (d, 2H, 2CH pyrazole, $J=7.1 \mathrm{~Hz}), 6.28,6.63(2 \mathrm{~d}, 2 \mathrm{H}, \mathrm{CH}=\mathrm{CH}, J=8.5 \mathrm{~Hz}), 7.05-8.13$ (m, 12H, Ar-H), $10.41\left(\mathrm{~s}, 1 \mathrm{H}, \mathrm{SO}_{2} \mathrm{NH}\right), 12.00(\mathrm{~s}, 1 \mathrm{H}, \mathrm{NH})$. ${ }^{13} \mathrm{C}-\mathrm{NMR}: 56.13,56.16,95.7,104.3,110.5,110.6,111.4(2)$, $121.8,122.0,124.7(2), 127.9,129.3(2), 131.5,132.1(2), 135.0$, 138.7, 142.5, 144.6, 145.7, 149.1, 152.8, 189.7. MS m/z (\%): 504 $\left(\mathrm{M}^{+}\right)$(16.38), 169 (100). Anal. Calcd for $\mathrm{C}_{26} \mathrm{H}_{24} \mathrm{~N}_{4} \mathrm{O}_{5} \mathrm{~S}$ (504): C, 61.89; H, 4.79; N, 11.10. Found: C, 61.56; H, 5.28; N, 11.41. Z-4-(3-(3,4-Dimethoxyphenyl)-3-oxoprop-1-enylamino)- $N$ (thiazol-2-yl)-benzenesulfonamide (9)

Yield, 83\%; mp $260.3^{\circ} \mathrm{C}$. IR: 3151 (NH), 3101 (arom.), 2971, 2929, 2860 (aliph.), 1635 (CO), 1589 (CN), 1375, $1168\left(\mathrm{SO}_{2}\right)$. ${ }^{1} \mathrm{H}-\mathrm{NMR}: 3.83\left(\mathrm{~s}, 6 \mathrm{H}, 2 \mathrm{OCH}_{3}\right), 6.22,6.58(2 \mathrm{~d}, 2 \mathrm{H}, \mathrm{CH}=\mathrm{CH}$, $J=8.6 \mathrm{~Hz}), 6.80-8.14(\mathrm{~m}, 9 \mathrm{H}, \mathrm{Ar}-\mathrm{H}+2 \mathrm{CH}$ thiazole), 10.30 (s, $\left.1 \mathrm{H}, \mathrm{SO}_{2} \mathrm{NH}\right), 12.02$ (s, $\left.1 \mathrm{H}, \mathrm{NH}\right) .{ }^{13} \mathrm{C}-\mathrm{NMR}: 56.11,56.13,95.2$, $108.5,110.5,110.6,111.3,111.4,121.9,124.9,128.2,131.5(2)$, 136.4, 142.7, 144.7, 149.0, 152.7, 169.1, 189.5. MS m/z (\%): 445 $\left(\mathrm{M}^{+}\right.$) (29.11), 93 (100). Anal. Calcd for $\mathrm{C}_{20} \mathrm{H}_{10} \mathrm{~N}_{3} \mathrm{O}_{5} \mathrm{~S}_{2}$ (445): C, 53.92; H, 4.30; N, 9.43. Found: C, 53.59; H, 3.95; N, 9.14.

Z-4-(3-(3,4-Dimethoxyphenyl)-3-oxoprop-1-enylamino)- $N$ (5-methyl-1,3,4-thiadiazol-2-yl)benzenesulfonamide (10)

Yield, 80\%; mp $186.9^{\circ} \mathrm{C}$. IR: 3153 (NH), 3055 (arom.), 2926, 2839, (aliph.), 1635 (CO), 1589 (CN), 1375, $1141\left(\mathrm{SO}_{2}\right)$. ${ }^{1} \mathrm{H}-\mathrm{NMR}: 2.44$ (s, 3H, $\mathrm{CH}_{3}$ ), $3.83\left(\mathrm{~s}, 6 \mathrm{H}, 2 \mathrm{OCH}_{3}\right), 6.24,6.59$ $(2 \mathrm{~d}, 2 \mathrm{H}, \mathrm{CH}=\mathrm{CH}, J=8.5 \mathrm{~Hz}), 7.04-8.13(\mathrm{~m}, 7 \mathrm{H}, \mathrm{Ar}-\mathrm{H}), 10.31$ (s, $\left.1 \mathrm{H}, \mathrm{SO}_{2} \mathrm{NH}\right), 12.02$ (s, $\left.1 \mathrm{H}, \mathrm{NH}\right) .{ }^{13} \mathrm{C}-\mathrm{NMR}: 16.5,55.1(2)$, $95.4,110.5,110.6,111.3,111.4,121.7,121.9,128.1,131.5(2)$, $144.1,145.1,149.1,152.5,154.8,168.2,186.7 . \mathrm{MS} m / z$ (\%): 460 $\left(\mathrm{M}^{+}\right.$) (8.75), 124 (100). Anal. Calcd for $\mathrm{C}_{20} \mathrm{H}_{20} \mathrm{~N}_{4} \mathrm{O}_{5} \mathrm{~S}_{2}$ (460.53): C, 52.16; H, 4.38; N, 12.17. Found: C, 52.42; H, 4.09; N, 12.39.

$\mathrm{Z}$-4-(3-(3,4-Dimethoxyphenyl)-3-oxoprop-1-enylamino)- $\mathrm{N}$ (pyridin-2-yl)benzenesulfonamide (11)

Yield, 79\%; mp $240.4^{\circ} \mathrm{C}$. IR: 3431 (NH), 3101 (arom.), 2931, 2835 (aliph.), 1635 (CO), 1589 (CN), 1394, $1151\left(\mathrm{SO}_{2}\right)$. ${ }^{1} \mathrm{H}-\mathrm{NMR}: 3.83\left(\mathrm{~s}, 6 \mathrm{H}, 2 \mathrm{OCH}_{3}\right), 6.22,6.58(2 \mathrm{~d}, 2 \mathrm{H}, \mathrm{CH}=\mathrm{CH}$, $J=8.5 \mathrm{~Hz}), 6.87-8.13(\mathrm{~m}, 11 \mathrm{H}, \mathrm{Ar}-\mathrm{H}), 10.30\left(\mathrm{~s}, 1 \mathrm{H}, \mathrm{SO}_{2} \mathrm{NH}\right)$, 12.00 (s, 1H, NH). ${ }^{13} \mathrm{C}-\mathrm{NMR}: 56.1(2), 95.4,110.5,110.6,111.3$, $113.8,113.9,115.2,121.8,121.9,129.2,129.3(2), 135.6,140.6$, 144.1, 149.0, 152.5, 152.7, 152.7, 189.6. MS $m / z(\%): 439\left(\mathrm{M}^{+}\right)$ (23.19), 77 (100). Anal. Calcd for $\mathrm{C}_{22} \mathrm{H}_{21} \mathrm{~N}_{3} \mathrm{O}_{5} \mathrm{~S}$ (439): C, 60.12; H, 4.82; N, 9.56. Found: C, 60.39; H, 4.49; N, 9.21.
Z-4-(3-(3,4-Dimethoxyphenyl)-3-oxoprop-1-enylamino)- $N$ (pyrimidin-2-yl)benzenesulfonamide (12)

Yield, 83\%; mp $272.5^{\circ} \mathrm{C}$. IR: 3221 (NH), 3088 (arom.), 2939, 2875, 2823 (aliph.), 1653 (CO), 1577 (CN), 1375, 1184 $\left(\mathrm{SO}_{2}\right) .{ }^{1} \mathrm{H}-\mathrm{NMR}: 3.83\left(\mathrm{~s}, 6 \mathrm{H}, 2 \mathrm{OCH}_{3}\right), 6.24,6.59(2 \mathrm{~d}, 2 \mathrm{H}$, $\mathrm{CH}=\mathrm{CH}, J=8.5 \mathrm{~Hz}), 7.04-8.51(\mathrm{~m}, 10 \mathrm{H}, \mathrm{Ar}-\mathrm{H}), 10.34(\mathrm{~s}, 1 \mathrm{H}$, $\left.\mathrm{SO}_{2} \mathrm{NH}\right), 12.00(\mathrm{~s}, 1 \mathrm{H}, \mathrm{NH}) .{ }^{13} \mathrm{C}-\mathrm{NMR}: 56.5(2), 95.6,110.4$, $110.6,111.3,111.4,115.0,121.8,121.9,130.0,131.4(2), 142.5$, $144.5,149.0,152.5,157.4,158.8,172.5,189.6$. MS $m / z(\%): 440$ $\left(\mathrm{M}^{+}\right)$(1.85), 79 (100). Anal. Calcd for $\mathrm{C}_{21} \mathrm{H}_{20} \mathrm{~N}_{4} \mathrm{O}_{5} \mathrm{~S}$ (440): C, 57.26; H, 4.58; N, 12.72. Found: C, 56.88; H, 4.22; N, 12.44 .

$\mathrm{Z}$-4-(3-(3,4-Dimethoxyphenyl)-3-oxoprop-1-enylamino)- $\mathrm{N}$ (4-methylpyrimidin-2-yl)benzenesulfonamide (13)

Yield, 90\%; mp $245.2^{\circ} \mathrm{C}$. IR: $3410(\mathrm{NH}), 3100$ (arom.), 2958, 2923, 2836 (aliph.), 1635 (CO), 1589 (CN), 1338, 1151 $\left(\mathrm{SO}_{2}\right) .{ }^{1} \mathrm{H}-\mathrm{NMR}: 2.30\left(\mathrm{~s}, 3 \mathrm{H}, \mathrm{CH}_{3}\right), 3.83\left(\mathrm{~s}, 6 \mathrm{H}, 2 \mathrm{OCH}_{3}\right), 6.23$, $6.59(2 \mathrm{~d}, 2 \mathrm{H}, \mathrm{CH}=\mathrm{CH}, J=8.6 \mathrm{~Hz}), 6.89-8.32(\mathrm{~m}, 9 \mathrm{H}, \mathrm{Ar}-\mathrm{H})$, $10.33\left(\mathrm{~s}, 1 \mathrm{H}, \mathrm{SO}_{2} \mathrm{NH}\right), 12.01(\mathrm{~s}, 1 \mathrm{H}, \mathrm{NH}) .{ }^{13} \mathrm{C}-\mathrm{NMR}: 23.7$, $56.11,56.12,95.5,110.5,110.6,111.3,112.5(2), 121.8,121.9$, $130.3,131.5(2), 142.5,145.4,149.0,152.8,157.0,157.9,168.7$, 189.6. MS m/z (\%): $454\left(\mathrm{M}^{+}\right)$(24.28), 93 (100). Anal. Calcd for $\mathrm{C}_{22} \mathrm{H}_{22} \mathrm{~N}_{4} \mathrm{O}_{5} \mathrm{~S}$ (454): C, 58.14; $\mathrm{H}, 4.88 ; \mathrm{N}, 12.33$. Found: $\mathrm{C}$, 58.48; H, 4.56; N, 12.03 .

Z-4-(3-(3,4-Dimethoxyphenyl)-3-oxoprop-1-enylamino)- $N$ (4,6-dimethylpyrimidin-2-yl)benzenesulfonamide (14)

Yield, 92\%; mp $276.5^{\circ} \mathrm{C}$. IR: 3220 (NH), 3056 (arom.), 2939, 2843 (aliph.), 1635 (CO), 1587 (CN), 1340, $1149\left(\mathrm{SO}_{2}\right)$. ${ }^{1} \mathrm{H}-\mathrm{NMR}: 2.24\left(\mathrm{~s}, 6 \mathrm{H}, 2 \mathrm{CH}_{3}\right), 3.83\left(\mathrm{~s}, 6 \mathrm{H}, 2 \mathrm{OCH}_{3}\right), 5.96$ (s, $1 \mathrm{H}, \mathrm{CH}$ pyrimidine), $6.55,6.76(2 \mathrm{~d}, 2 \mathrm{H}, \mathrm{CH}=\mathrm{CH}, J=8.5 \mathrm{~Hz})$, 7.04-8.11 (m, 7H, Ar-H), 10.31 (s, $\left.1 \mathrm{H}, \mathrm{SO}_{2} \mathrm{NH}\right), 12.01(\mathrm{~s}, 1 \mathrm{H}$, $\mathrm{NH}) .{ }^{13} \mathrm{C}-\mathrm{NMR}:$ 23.5(2), 56.1(2), 96.4, 108.2, 110.6, 111.4, $112.3(2), 114.6,115.5,130.5,130.7(2), 142.8,146.7,149.1$, 152.6, 163.8(2), 167.7, 184.2. MS $m / z(\%): 468\left(\mathrm{M}^{+}\right)(22.17)$, 164 (100). Anal. Calcd for $\mathrm{C}_{23} \mathrm{H}_{24} \mathrm{~N}_{4} \mathrm{O}_{5} \mathrm{~S}$ (468): C, 58.96; $\mathrm{H}$, 5.16; N, 11.96. Found: C, 58.67; H, 5.44; N, 12.33 .

Z-4-(3-(3,4-Dimethoxyphenyl)-3-oxoprop-1-enylamino)- $N$ (2,6-dimethylpyrimidin-4-yl)benzenesulfonamide (15)

Yield, 87\%; mp $243.3^{\circ} \mathrm{C}$. IR: 3415 (NH), 3072 (arom.), 2935, 2839 (aliph.), 1637 (CO), 1589 (CN), 1348, $1149\left(\mathrm{SO}_{2}\right)$. ${ }^{1} \mathrm{H}-\mathrm{NMR}: 2.51\left(\mathrm{~s}, 6 \mathrm{H}, 2 \mathrm{CH}_{3}\right), 3.82\left(\mathrm{~s}, 6 \mathrm{H}, 2 \mathrm{OCH}_{3}\right), 6.22,6.59$ $(2 \mathrm{~d}, 2 \mathrm{H}, \mathrm{CH}=\mathrm{CH}, J=8.5 \mathrm{~Hz}), 7.01-8.14(\mathrm{~m}, 8 \mathrm{H}, \mathrm{Ar}-\mathrm{H}), 10.34$ (s, $\left.1 \mathrm{H}, \mathrm{SO}_{2} \mathrm{NH}\right), 12.00(\mathrm{~s}, 1 \mathrm{H}, \mathrm{NH}) .{ }^{13} \mathrm{C}-\mathrm{NMR}: 19.0,22.3$, 56.1(2), 95.7, 100.6, 110.5, 110.6, 111.3, 111.4, 121.8, 121.9, $127.7,129.2$, 130.3, 142.3, 146.5, 149.0, 152.5, 152.8, 162.6, 163.0, 186.8. MS m/z (\%): $468\left(\mathrm{M}^{+}\right)$(5.63), 89 (100). Anal. Calcd for $\mathrm{C}_{23} \mathrm{H}_{24} \mathrm{~N}_{4} \mathrm{O}_{5} \mathrm{~S}$ (468): C, 58.96; H, 5.16; N, 11.96 . Found: C, 59.32; H, 4.87; N, 11.63.

Z-4-(3-(3,4-Dimethoxyphenyl)-3-oxoprop-1-enylamino)- $N$ (5-methoxylpyrimidin-2-yl)benzenesulfonamide (16)

Yield, 81\%; mp 216.7 ${ }^{\circ}$. IR: 3373 (NH), 3101 (arom.), 2837, 2735 (aliph.), 1635 (CO), 1591 (CN), 1375, $1153\left(\mathrm{SO}_{2}\right)$. ${ }^{1} \mathrm{H}-\mathrm{NMR}: 3.78\left(\mathrm{~s}, 3 \mathrm{H}, \mathrm{OCH}_{3}\right), 3.83\left(\mathrm{~s}, 6 \mathrm{H}, 2 \mathrm{OCH}_{3}\right), 6.23,6.59$ $(2 \mathrm{~d}, 2 \mathrm{H}, \mathrm{CH}=\mathrm{CH}, J=8.5 \mathrm{~Hz}), 7.02-8.30(\mathrm{~m}, 9 \mathrm{H}, \mathrm{Ar}-\mathrm{H}), 10.35$ (s, $\left.1 \mathrm{H}, \mathrm{SO}_{2} \mathrm{NH}\right), 12.02$ (s, $\left.1 \mathrm{H}, \mathrm{NH}\right) .{ }^{13} \mathrm{C}-\mathrm{NMR}: 55.9,56.6,56.7$, 95.6, 110.5, 110.6, 111.3, 111.4, 121.8, 125.7, 129.9, 130.1(2), 142.5(2), 144.0, 145.0, 145.4, 149.8, 152.5, 172.5, 186.8. MS m/z (\%): $470\left(\mathrm{M}^{+}\right)$(33.82), 123 (100). Anal. Calcd for $\mathrm{C}_{22} \mathrm{H}_{22} \mathrm{~N}_{4} \mathrm{O}_{6} \mathrm{~S}$ (470): C, 56.16; H, 4.71; N, 11.91. Found: C, 55.79; H, 4.47; N, 12.25 . 
Z-4-(3-(3,4-Dimethoxyphenyl)-3-oxoprop-1-enylamino)- $N$ (2,6-dimethoxylpyrimidin-4-yl)benzenesulfonamide (17)

Yield, 86\%; mp $201.3^{\circ} \mathrm{C}$. IR: 3134 (NH), 3061 (arom.), 2902, 2835 (aliph.), 1637 (CO), 1589 (CN), 1363, $1151\left(\mathrm{SO}_{2}\right)$. ${ }^{1} \mathrm{H}-\mathrm{NMR}: 3.78,3.79\left(2 \mathrm{~s}, 6 \mathrm{H}, 2 \mathrm{OCH}_{3}\right.$ pyrimidine), 3.83 (s, $\left.6 \mathrm{H}, 2 \mathrm{OCH}_{3}\right), 5.96(\mathrm{~s}, 1 \mathrm{H}, \mathrm{CH}$ pyrimidine $), 6.24,6.58(2 \mathrm{~d}, 2 \mathrm{H}$, $\mathrm{CH}=\mathrm{CH}, J=8.6 \mathrm{~Hz}), 7.02-8.12(\mathrm{~m}, 7 \mathrm{H}, \mathrm{Ar}-\mathrm{H}), 10.37$ (s, 1H, $\left.\mathrm{SO}_{2} \mathrm{NH}\right), 12.01(\mathrm{~s}, 1 \mathrm{H}, \mathrm{NH}) .{ }^{13} \mathrm{C}-\mathrm{NMR}: 54.2,54.9,55.8,55.9$, $84.9,95.8,110.5,110.6,111.4(2), 121.8,121.9,129.6,129.8(2)$, $142.4,145.8,149.1,152.8,160.3,164.7,172.5,189.6$. MS m/z (\%): $500\left(\mathrm{M}^{+}\right)$(19.34), 152 (100). Anal. Calcd for $\mathrm{C}_{23} \mathrm{H}_{24} \mathrm{~N}_{4} \mathrm{O}_{7} \mathrm{~S}$ (500): C, 55.19; H, 4.83; N, 11.19. Found: C, 55.52; H, 4.57; N, 10.90 .

Z-4-(3-(3,4-Dimethoxyphenyl)-3-oxoprop-1-enylamino)- $N$ (5,6-dimethoxylpyrimidin-4-yl)benzenesulfonamide (18)

Yield, 80\%; mp 244.1 ${ }^{\circ} \mathrm{C}$. IR: 3189 (NH), 3082 (arom.), 2941, 2839 (aliph.), 1631 (CO), 1589 (CN), 1375, $1161\left(\mathrm{SO}_{2}\right)$. ${ }^{1} \mathrm{H}-\mathrm{NMR}: 3.70,3.90\left(2 \mathrm{~s}, 6 \mathrm{H}, 2 \mathrm{OCH}_{3}\right.$ pyrimidine $), 3.83(\mathrm{~s}, 6 \mathrm{H}$, $\left.2 \mathrm{OCH}_{3}\right), 6.20,6.60(2 \mathrm{~d}, 2 \mathrm{H}, \mathrm{CH}=\mathrm{CH}, J=8.5 \mathrm{~Hz}), 7.06-7.94$ (m, 7H, Ar- $\mathrm{H}), 8.12$ (s, 1H, CH pyrimidine), $10.30(\mathrm{~s}, 1 \mathrm{H}$, $\left.\mathrm{SO}_{2} \mathrm{NH}\right), 12.00$ (s, $\left.1 \mathrm{H}, \mathrm{NH}\right) .{ }^{13} \mathrm{C}-\mathrm{NMR}: 54.5,55.9,56.1,56.5$, $95.6,110.5,110.6,111.4(2), 121.8,121.9,127.6,130.2(2), 131.5$, $142.5,144.0,149.1,150.9,152.6(2), 162.0,189.6$. MS $\mathrm{m} / z(\%)$ : $500\left(\mathrm{M}^{+}\right)$(5.84), 151 (100). Anal. Calcd for $\mathrm{C}_{23} \mathrm{H}_{24} \mathrm{~N}_{4} \mathrm{O}_{7} \mathrm{~S}(500)$ : C, 55.19; H, 4.83; N, 11.19. Found: C, 54.92; H, 4.50; N, 11.48. Z-4-(3-(3,4-Dimethoxyphenyl)-3-oxoprop-1-enylamino)- $N$ (1H-indazol-6-yl)benzenesulfonamide (19)

Yield, 89\%; mp $252.4^{\circ} \mathrm{C}$. IR: 3346 (NH), 3100 (arom.), 2966, 2933, 2839 (aliph.), 1627 (CO), 1589 (CN), 1350, 1157 $\left(\mathrm{SO}_{2}\right) .{ }^{1} \mathrm{H}-\mathrm{NMR}: 3.82\left(\mathrm{~s}, 6 \mathrm{H}, 2 \mathrm{OCH}_{3}\right), 6.18,6.60(2 \mathrm{~d}, 2 \mathrm{H}$, $\mathrm{CH}=\mathrm{CH}, J=8.6 \mathrm{~Hz}), 6.95-8.08(\mathrm{~m}, 10 \mathrm{H}, \mathrm{Ar}-\mathrm{H}), 8.13(\mathrm{~s}, 1 \mathrm{H}$, $\mathrm{CH}$ pyrazole), 10.32 (s, $\left.1 \mathrm{H}, \mathrm{SO}_{2} \mathrm{NH}\right), 12.00$ (s, 1H, NH), 12.8 (s, 1H, NH pyrazole). ${ }^{13} \mathrm{C}-\mathrm{NMR}: 56.5(2), 95.6,100.3,110.4$, $110.6,111.3,115.3(2), 116.2,120.2,121.7,129.3,131.4(2)$, $132.2,140.7,142.4,143.8,144.4,149.0,152.8,186.8 . \mathrm{MS} \mathrm{m} / \mathrm{z}$ (\%): 478( $\left.\mathrm{M}^{+}\right)$(14.84), 116 (100). Anal. Calcd for $\mathrm{C}_{24} \mathrm{H}_{22} \mathrm{~N}_{4} \mathrm{O}_{5} \mathrm{~S}$ (478): C, 60.24; H, 4.63; N, 11.71. Found: C, 60.53; H, 4.31; N, 11.98 .

\section{In Vitro Anticancer Evaluation}

\section{Chemicals and Supplies}

MTT and DMSO were purchased from Sigma-Aldrich. Dulbecco's modified Eagle's medium (DMEM)/high glucose, DMEM/F12, fetal bovine serum (FBS), L-glutamine, and penicillin-streptomycin were obtained from Gibco Inc. (NY, U.S.A.).

\section{Cell Lines}

Four tumor cell lines were utilized in this study, namely the HeLa, HepG2, Daoy, and the HT-29 cells. These cell lines were obtained from American Type Culture Collection (Manassas, VA, U.S.A.). HeLa, HepG2, and HT-29 cells were cultured in DMEM/high glucose supplemented with $10 \% \mathrm{FBS}, 2 \mathrm{~mm}$ L-glutamine, and 1\% penicillin-streptomycin. Daoy cells were cultured in DMEM/F12 supplemented with $10 \%$ FBS, 2 mM L-glutamine, and 1\% penicillin-streptomycin.

Methods

Growth Inhibitory Activity of the New Compounds Screened by MTT Assay

The antiproliferative activity of the new compounds was assessed at the Cell Culture Laboratory, College of Pharmacy, King Saud University, in a primary four cell lines-one concentration $(25 \mu \mathrm{g} / \mathrm{mL})$ anticancer assay by using the aforemen- tioned cell lines. The cytotoxicity of the newly synthesized compounds was determined by testing the capacity of the reducing enzymes present in the viable cells to convert MTT to formazan crystals as previously reported. ${ }^{43)}$ Briefly, cells were seeded in complete medium, at a density of $2 \times 10^{4}$ cells/ well, into 96-well microtiter plates and incubated at $37^{\circ} \mathrm{C}$ in a humidified atmosphere with $5 \% \mathrm{CO}_{2}$, for $24 \mathrm{~h}$. The complete medium was then changed to serum-free medium (SFM), containing the test compounds $(25 \mu \mathrm{g} / \mathrm{mL})$, or an equivalent volume of solvent (DMSO), as control. After incubation, the SFM in the control and test wells was replaced by MTT $(100 \mu \mathrm{L} /$ well; $0.5 \mathrm{mg} / \mathrm{mL})$ in phosphate-buffered saline (PBS) and incubated at $37^{\circ} \mathrm{C}$ for an additional $3 \mathrm{~h}$. The MTT solution was removed and the purple formazan crystals formed at the bottom of the wells were dissolved using isopropyl alcohol $(100 \mu \mathrm{L} / w e l l)$, and the plate was further incubated for $2 \mathrm{~h}$ at room temperature, with constant agitation. The plate was read in a microplate reader (ELX 800; Bio-Tek Instruments, Winooski, VT, U.S.A.), and the absorbance at $549 \mathrm{~nm}$ recorded.

The dose response curves of the compounds exhibiting $\geq 50 \%$ inhibition in the aforementioned one-dose prescreening assay for each cell line were established using the following concentrations: $1.56,3.125,6.25,12.5,25$, and $50 \mu \mathrm{g} /$ $\mathrm{mL}$. The concentration effecting $\mathrm{IC}_{50}$ was calculated. The cytotoxic activity of the well-known antitumor drug dasatinib, a potent multi-targeted inhibitor of BCR-ABL and SRC family kinases, ${ }^{44)}$ was determined at the same concentrations of the tested compounds against the four cell lines, and utilized as a standard for comparative purposes.

In Vitro VEGFR-2 Inhibition Assay HeLa cells $\left(1.2-1.8 \times 10^{4}\right.$ cells/well) were cultured in a volume of $200 \mu \mathrm{L} /$ well $(100 \mu \mathrm{L}$ of complete growth medium $+100 \mu \mathrm{L}$ of the tested compounds) in a 96-well plate for $18-24 \mathrm{~h}$ before the VEGFR-2 enzyme assay was performed. ${ }^{45}$

Molecular Docking Molecular docking studies were performed on an Intel Pentium $1.6 \mathrm{GHz}$ processor, $512 \mathrm{MB}$ memory with Windows XP operating system using Molecular Operating Environment (MOE, 10.2008) software. All the minimizations were performed with MOE until an RMSD gradient of $0.05 \mathrm{kcalmol}^{-1} \AA^{-1}$ with MMFF94X force field, and the partial charges were automatically calculated. The X-ray crystallographic structure of VEGFR-2 complexed with sunitinib was obtained from the protein data bank (PDB ID: 4AGD). The protein was prepared for docking studies in the following way: (i) the co-crystallized ligand molecule was removed from the enzyme active site; (ii) hydrogen atoms were added to the structure with their standard geometry; (iii) MOE Alpha Site Finder was used to search for the active site in the protein structure and to generate dummy atoms from the obtained alpha spheres; (iv) the obtained model was then used in predicting ligand-protein interactions at the active site.

\section{Conclusion}

A novel series of benzenesulfonamide derivatives bearing the 3,4-dimethoxyphenyl moiety were synthesized and evaluated for their cytotoxic activity against four different cell lines. Four compounds $(4,10,16,19)$ showed good cytotoxic activity when compared to dasatinib (reference drug). The same compounds were evaluated for their in vitro ability to inhibit VEGFR-2. Compounds $\mathbf{1 0}$ and $\mathbf{1 9}$ were more active than dasatinib as VEGFR-2 inhibitors. Structure-activity 
relationship favors heterocyclic sulfonamide derivatives and, in particular, methyl thiadiazole derivative $\mathbf{1 0}$ and benzopyrazole derivative 19. Molecular docking on the active site of VEGFR-2 for compound 19 exhibited good fitting and comparable amino acid interactions to those displayed by sunitinib.

Acknowledgment This project was funded by the National Plan for Science, Technology and Innovation (MAARIFAH), King Abdulaziz City for Science and Technology, Kingdom of Saudi Arabia, Award Number (13-MED 997-02).

Conflict of Interest The authors declare no conflict of interest

\section{References}

1) Jemal A., Bray F., Center M. M., Ferlay J., Ward E. F. D., Forman D., Cancer J. Clin., 61, 69-90 (2011).

2) Thun M. J., DeLancey J. O., Center M. M., Jemal A., Ward E. M., Carcinogenesis, 31, 100-110 (2010).

3) Heffeter P., Jakupec M. A., Körner W., Wild S., von Keyserlingk N. G., Elbling L., Zorbas H., Korynevska A., Knasmüller S., Sutterlüty H., Micksche M., Keppler B. K., Berger W., Biochem. Pharmacol., 71, 426-440 (2006).

4) Zwick E., Bange J., Ullrich A., Endocr. Relat. Cancer, 8, 161-173 (2001)

5) Peng F.-W., Xuan J., Wu T.-T., Xue J.-Y., Ren Z.-W., Liu D.-K., Wang X.-Q., Chen X.-H., Zhang J.-W., Xu Y.-G., Shi L., Eur. J. Med. Chem., 109, 1-12 (2016).

6) Sun L., Tran N., Liang C., Tang F., Rice A., Schreck R., Waltz K. Shawver L. K., McMahon G., Tang C., J. Med. Chem., 42, 51205130 (1999).

7) Kubo K., Shimizu T., Ohyama S., Murooka H., Iwai A., Nakamura K., Hasegawa K., Kobayashi Y., Takahashi N., Takahashi K., Kato S., Izawa T., Isoe T., J. Med. Chem., 48, 1359-1366 (2005).

8) Hennequin L. F., Stokes E. S. E., Thomas A. P., Johnstone C., Plé P. A., Ogilvie D. J., Dukes M., Wedge S. R., Kendrew J., Curwen J. O., J. Med. Chem., 45, 1300-1312 (2002).

9) Tripathy R., Reiboldt A., Messina P. A., Iqbal M., Singh J., Bacon E. R., Angeles T. S., Yang S. X., Albom M. S., Robinson C., Chang H., Ruggeri B. A., Mallamo J. P., Bioorg. Med. Chem. Lett., 16, 2158-2162 (2006).

10) Munchhof M. J., Beebe J. S., Casavant J. M., Cooper B. A., Doty J. L., Higdon R. C., Hillerman S. M., Soderstrom C. I., Knauth E. A., Marx M. A., Rossi A. M. K., Sobolov S. B., Sun J., Bioorg. Med. Chem. Lett., 14, 21-24 (2004).

11) Mendel D. B., Laird A. D., Smolich B. D., Blake R. A., Liang C., Hannah A. L., Shaheen R. M., Ellis L. M., Weitman S., Shawver L. K., Cherrington J. M., Anticancer Drug Des., 15, 29-41 (2000).

12) Scott R. W., Neville S. N., Urbina A., Camp D., Stankovic N., Org. Process Res. Dev., 10, 296-303 (2006).

13) Semenza G. L., N. Engl. J. Med., 358, 2066-2067 (2008).

14) Hicklin D. J., Ellis L. M., J. Clin. Oncol., 23, 1011-1027 (2005).

15) Ferrara N., Endocr. Rev., 25, 581-611 (2004).

16) Guo S., Colbert L. S., Fuller M., Zhang Y., Gonzalez-Perez R. R., Biochim. Biophys. Acta, 1806, 108-121 (2010).

17) Partanen T. A., Alitalo K., Miettinen M., Cancer, 86, 2406-2412 (1999).

18) Folkman J., N. Engl. J. Med., 285, 1182-1186 (1971).

19) Jain R. K., Duda D. G., Willett C. G., Sahani D. V., Zhu A. X., Loeffler J. S., Batchelor T. T., Sorensen A. G., Nat. Rev. Clin. Oncol., 6, 327-338 (2009).

20) Baka S., Clamp A. R., Jayson G. C., Expert Opin. Ther. Targets, 10, 867-876 (2006).

21) Gupta K., Zhang J., Postgrad. Med. J., 81, 236-242 (2005).
22) Sternberg C. N., Davis I. D., Mardiak J., Szczylik C., Lee E., Wagstaff J., Barrios C. H., Salman P., Gladkov O. A., Kavina A., Zarbá J. J., Chen M., McCann L., Pandite L., Roychowdhury D. F., Hawkins R. E., J. Clin. Oncol., 28, 1061-1068 (2010).

23) van der Graaf W. T., Blay J.-Y., Chawla S. P., Kim D.-W., BuiNguyen B., Casali P. G., Schöffski P., Aglietta M., Staddon A. P., Beppu Y., Le Cesne A., Gelderblom H., Judson I. R., Araki N., Ouali M., Marreaud S., Hodge R., Dewji M. R., Coens C., Demetri G. D., Fletcher C. D., Dei Tos A. P., Hohenberger P.; EORTC Soft Tissue and Bone Sarcoma Group, PALETTE study group, Lancet, 379, 1879-1886 (2012).

24) Bukowski R. M., Yasothan U., Kirkpatrick P., Nat. Rev. Drug Discov., 9, 17-18 (2010).

25) Fukuoka K., Usuda J., Iwamoto Y., Fukumoto H., Nakamura T., Yoneda T., Narita N., Saijo N., Nishio K., Invest. New Drugs, 19, 219-227 (2001).

26) Supuran C. T., Scozzafava A., Expert Opin. Ther. Pat., 10, 575-600 (2000).

27) Payne J. E., Bonnefous C., Hassig C. A., Symons K. T., Guo X., Nguyen P.-M., Annable T., Wash P. L., Hoffman T. Z., Rao T. S., Shiau A. K., Malecha J. W., Noble S. A., Hager J. H., Smith N. D., Bioorg. Med. Chem. Lett., 18, 6093-6096 (2008).

28) Kawai M., BaMaung N. Y., Fidanze S. D., Erickson S. A., Tedrow J. S., Sanders W. J., Vasudevan A., Park C., Hutchins C., Comess K. M., Kalvin D., Wang J., Zhang Q., Lou P., Tucker-Garcia L., Bouska J., Bell R. L., Lesniewski R., Henkin J., Sheppard G. S., Bioorg. Med. Chem. Lett., 16, 3574-3577 (2006).

29) Supuran C. T., Scozzafava A., Expert Opin. Ther. Pat., 12, 217-242 (2002).

30) Villar R., Encio I., Migliaccio M., Gil M. J., Martinez-Merino V., Bioorg. Med. Chem., 12, 963-968 (2004).

31) Huang S., Connolly P. J., Lin R., Emanuel S., Middleton S. A., Bioorg. Med. Chem. Lett., 16, 3639-3641 (2006).

32) Hande K. R., Hagey A., Berlin J., Cai Y., Meek K., Kobayashi H., Lockhart A. C., Medina D., Sosman J., Gordon G. B., Rothenberg M. L., Clin. Cancer Res., 12, 2834-2840 (2006).

33) Nakamura K., Yamamoto A., Kamishohara M., Takahashi K., Taguchi E., Miura T., Kubo K., Shibuya M., Isoe T., Mol. Cancer Ther., 3, 1639-1649 (2004).

34) Al-Dosari M. S., Ghorab M. M., Al-Said M. S., Nissan Y. M., Chem. Pharm. Bull., 61, 50-58 (2013).

35) Al-Said M. S., Ghorab M. M., Nissan Y. M., Chem. Cent. J., 6, 64 (2012).

36) Ghorab M. M., Ragab F. A., Heiba H. I., Agha H. M., Nissan Y. M., Arch. Pharm. Res., 35, 59-68 (2012).

37) Al-Dosari M. S., Ghorab M. M., Alsaid M. S., Nissan Y. M., Ahmed A. B., Eur. J. Med. Chem., 69, 373-383 (2013).

38) Ghorab M. M., Ceruso M., Alsaid M. S., Nissan Y. M., Arafa R. K., Supuran C. T., Eur. J. Med. Chem., 87, 186-196 (2014).

39) Ghorab M. M., Alsaid M. S., Ceruso M., Nissan Y. M., Supuran C. T., Bioorg. Med. Chem., 22, 3684-3695 (2014).

40) Ghorab M. M., Alsaisd M. S., Nissan Y. M., Acta Pol. Pharm., 72, 65-78 (2015).

41) Ghorab M. M., Alsaid M. S., Al-Dosari M. S., Nissan Y. M., AlMishari A. A., Chem. Cent. J., 10, 18 (2016).

42) Wan J.-P., Cao S., Liu Y., J. Org. Chem., 80, 9028-9033 (2015).

43) Al-Salahi M. M. R., Ashour A. E., Alswaidan I., Asian J. Chem., 26, 2173-2176 (2014).

44) Lombardo L. J., Lee F. Y., Chen P., Norris D., Barrish J. C., Behnia K., Castaneda S., Cornelius L. A. M., Das J., Doweyko A. M., Fairchild C., Hunt J. T., Inigo I., Johnston K., Kamath A., Kan D., Klei H., Marathe P., Pang S., Peterson R., Pitt S., Schieven G. L., Schmidt R. J., Tokarski J., Wen M.-L., Wityak J., Borzilleri R. M., J. Med. Chem., 47, 6658-6661 (2004).

45) Biovendor kit. Human VEGF-R2/KDR ELISA. Cat. No.: RBMS2019R. 